\title{
Mortalidad materna y factores de riesgo asociados. Estudio de casos y controles. Area metropolitana de Medellín. Octubre 1 de 1988 - septiembre 30 de 1989
}

\author{
Octavio Cataño*
}

RESUMEN: Estudio poblacional de casos y controles que se hizo para identificar los principales factores de riesgo que inciden en la Mortalidad Materna y conocer el peso real de cada uno de ellos, con el fin de orientar las acciones de control en forma efectiva y producir impacto en la reducción de la problemática en el Area Metropolitana de Medellín, Colombia. Se logró igualmente cuantificar y clasificar la mortalidad en las mujeres en edad reproductiva (12-49 años) y la Mortalidad Materna.

El estudio incluyó todas las muertes maternas producidas entre octubre de 1988 y septiembre de 1989 . Se tomaron cuatro controles por caso, la mitad de ellos institucionales y el resto en la población. Al estudiar los factores socioeconómicos se tomó como base la estratificación que divide el AMM en seis estratos y se obtuvieron tres estratos para el presente estudio. Además de los certificados de defunción, se tomaron los registros de egreso hospitalario por muerte de mujeres de edad reproductiva, los informes de necropsia, las historias clínicas y se hicieron visitas domiciliarias para entrevistar al familiar más cercano de la mujer fallecida. Para los controles también se hizo visita al familiar más próximo.

El subregistro encontrado fue del $52.8 \%$. La tasa de mortalidad de las mujeres en edad fértil fue de 15 por 10.000 y la tercera parte de estas muertes se debió a causas violentas y accidentales. La tasa de mortalidad materna fue de 7.2 por 10.000 nacidos vivos y es más de dos veces mayor que la informada oficialmente para 1988. Las causas de muerte materna en orden de frecuencia fueron la hipertensión asociada al embarazo, la infección y la hemorragia. El aborto puede considerarse como la segunda causa porque está englobada por la infección y la hemorragia.

Los factores socioeconómicos fueron los que presentaron mayor asociación con la Mortalidad Materna, y se destacan en ellos la baja escolaridad tanto de la mujer como de su compañero, el estrato socioeconómico bajo y la ausencia de apoyo afectivo para la mujer. Sus razones de disparidad fluctuaron entre 3.5 y 4.9. Entre los antecedentes personales y obstétricos se destacó el estado civil soltera con una razón de disparidad de 2.6. Entre los factores de riesgo del embarazo en el cual se produjo la muerte se encontró mayor fuerza de asociación con la hemorragia, aunque la hipertensión presentó una razón de disparidad de 10.0. Entre los factores de riesgo relacionados con la accesibilidad a los servicios de salud no se encontró asociación estadísticamente significativa.

La proporción de riesgo atribuible a los expuestos permitió determinar una evitabilidad de las muertes maternas entre el $61.5 \%$ y el $91 \%$. Se recomiendan intervenciones dentro del enfoque de riesgo para impactar los factores de riesgo específicos que se encontraron asociados con la Mortalidad Materna en el presente estudio.

PALABRAS CLAVES: Mortalidad materna, factores de riesgo.

SUMMARY: A community based case-control study was performed to identify the risk factors for Maternal Mortality (MM) in order to orientate control actions to diminish maternal deaths in the Metropolitan Area of Medellín, Colombia. Both Fertil Age Women Mortality and MM were cuantified and classified. All maternal deaths occurred during the study period were included (October 1988 - September 1989). A 4:1 ratio of controls to cases was taken, half the controls were sampled from the population and the rest were randomly sampled from hospitals. As the Metropolitan Area is already divided in six social strata, they were grouped in three strata to compare in this study. Sources of information were not only death certificates but reproductive age women hospital discharges, necropsy records, clinical histories and domiciliary visits to the next of kin of the deceased women. The next of kin of the controls were also interviewed.

The Reproductive Age Women Mortality Rate was 15.0 per 10.000, one third due to violence and accidents. The MM Rate was 7.2 per 10.000 live births, which was more than twice as high as the official rate in 1988, it was found to be $52.8 \%$ underestimated. The causes of maternal deaths were hypertensive disease of pregnancy, followed by infection and haemorrhage which encompass abortion, which was in fact the second cause of death.

The socioeconomic risk factors were found to be highly associated to MM: low level of education of both the woman and her partner, low socioeconomic stratum and the lack of affection support had the greatest strength of association having been their odds rations between 3.5 and 4.9. Personal and obstetrical antecedents were also considered, but only marital status was found to have statistically significant odds ratio $=2.6$ if to be single was considered to be a risk factor.

The risk factor of the last pregnancy were evaluated and haemorrhage was found to have odds ratio $=3.7$ with great strength of association. The odds ratio of hypertension was 10.0. The access to health services depend on some risk factors which were found not to be statistically significant.

The proportion of Attributable risk due only to the exposure evidenced MM to be preventable between $61.5 \%$ to $91 \%$. Risk approach interventions are recommended taking into account the risk factors found to be associated to Maternal deaths in the present study.

KEY WORDS: Maternal mortality, risk factors.

* Profesor de Medicina Preventiva y Salud Pública, Facultad de Medicina, Universidad de Antioquia. 


\section{Introducción}

La disminución de la Mortalidad Materna ha recibido especial prioridad por parte de la Organización Mundial de la Salud en los últimos decenios y muy especialmente a partir de la Reunión Interregional de Nairobi en 1985. Los gobiernos han apoyado las políticas y estrategias de allí derivadas y ahora incluyen la atención integral de la mujer dentro de los programas de salud.

La mujer en edad reproductiva, especialmente en los llamados países en desarrollo, está expuesta a muchos factores de riesgo que condicionan la morbimortalidad materna y perinatal. Si se mide la asociación entre varios "factores" y la morbilidad o la mortalidad materna, se puede predecir qué grupos de mujeres tienen mayor riesgo de sufrir una evolución desfavorable de su embarazo y se pueden, por lo tanto, plantear alternativas para evitar este resultado que afecta a la población en general y no sólo a la mujer como pudiera parecer en primera instancia.

El número de muertes maternas no es conocido con exactitud en los países desarrollados y mucho menos en los demás. La precisión que se logre en este indicador le permitirá a la Medicina Preventiva establecer acciones más precisas, teniendo en cuenta que son muertes evitables con el conocimiento y las tecnologías disponibles en el momento actual.

Muchos estudios sobre Mortalidad Materna son estudios institucionales que carecen de un grupo comparativo que pueda proporcionar un mayor significado a sus hallazgos. En los últimos años, los estudios de casos y controles se han hecho cada vez más corrientes en diferentes sectores de la investigación, pero son fundamentalmente pocos los dedicados a la Mortalidad Materna y cuando así ocurre, la mayoría de los que se presentan también son institucionales. Sin embargo, es perfectamente factible realizar estudios de casos y controles en la comunidad.

El Estudio Sectorial de Salud pone en claro que en Colombia la investigación descriptiva presenta predominio sobre los estudios explicativos, analíticos y básicos. Es decir, se da más importancia a conocer la magnitud del fenómeno que comprender los factores que lo determinan.

$\mathrm{El}$ presente estudio de casos y controles pretende contribuir a llenar el vacío que revelan estas carencias y llamar la atención para que otros investigadores hagan aportes en esta misma línea de trabajo.

\section{Propósito}

Identificar los principales factores de riesgo que inciden en la Mortalidad Materna y el conocimiento del peso real de cada uno de ellos en esta problemática, con el fin de orientar las acciones de control en forma más efectiva y producir impacto en la reducción de la mortalidad materna en el Area Metropolitana de Medellín, Colombia.

\section{Objetivos}

1. Cuantificar y clasificar la mortalidad en mujeres en edad reproductiva (12 a 49 años).

2. Cuantificar la Mortalidad Materna.

3. Caracterizar la Mortalidad Materna de acuerdo con las variables de persona y lugar.

4. Determinar la asociación entre Mortalidad Materna y los factores de riesgo, incluyendo aquellos relacionados con el estado socioeconómico, el entorno familiar y social y la contracepción.

5. Identificar los factores de riesgo que inciden en la Mortalidad Materna.

6. Establecer los aspectos fundamentales sobre los cuales se deben basar las intervenciones para la disminución de la Mortalidad Materna en el Area Metropolitana de Medellín.

\section{Materiales y métodos}

Se realizó un estudio poblacional de casos y controles que incluyó todas las muertes maternas ocurridas en el Area Metropolitana de Medellín (AMM), durante un período de un año, comprendido entre el 1o. de octubre de 1988 y el 30 de septiembre de 1989. La parte descriptiva del estudio permitió establecer la mortalidad de las mujeres en edad fértil (12-49 años) y la mortalidad materna en el área de estudio, la cual se pudo caracterizar de acuerdo con las variables de persona y lugar.

Se definió como caso toda muerte materna producida por causa obstétrica directa o indirecta y residente en el AMM durante el período en estudio. Se definió como control toda mujer viva, en edad fértil, residente en el AMM que hubiera terminado su embarazo por cualquier causa, independiente del tiempo de gestación, durante el período en estudio.

El área de estudio incluye a la ciudad de Medellín, capital del departamento de Antioquia y a otros nueve municipios que conforman el área metropolitana, con una densidad de 2.012 habitantes por $\mathrm{Km}^{2}$. La población estimada para el AMM fue de 2.343.614 habitantes para 1988, de los cuales el $28.7 \%$ correspondía a mujeres en edad fértil. La población del AMM concentra alrededor del $60 \%$ de la población del departamento de Antioquia. El 70.5\% de los nacimientos ocurridos en 1988 en el AMM se presentaron en la ciudad de Medellín.

Estratificación socioeconómica: El AMM de Medellín se encuentra estratificada con el fin de permitir mayor equidad en el cobro de los servicios públicos. Los criterios de estratificación tienen en cuenta la estructura física de la vivienda, el barrio o lugar donde está ubicada, la accesibilidad a los servicios públicos, los servicios comunales de que dispone y el ingreso por familia y por persona. Se han definido seis estratos así: bajo-bajo, bajo, medio-bajo, medio-medio, medio-alto y alto. La ciudad de Medellín cuenta con 16 divisiones territoriales denominadas comunas, en el $85.7 \%$ de las cuales predominan los estratos bajo y mediobajo.

Para los fines del presente estudio, tanto los casos como los controles pudieron ser ubicados en sus respectivos estratos. Sin embargo, para el análisis, los estratos se agruparon en tres, así:

- Estrato bajo: incluye los estratos bajo y bajo-bajo.

- Estrato medio: incluye los estratos medio-bajo y mediomedio

- Estrato alto: incluye los estratos medio-alto y alto.

Muestra: Aunque se tomaron todos los casos ocurridos durante el período en estudio, se calculó el tamaño de muestra requerido para un estudio de casos y controles, de acuerdo con los cuatro parámetros básicos definidos por Schlesseman 
(1) y se encontró que se necesitaba por lo menos 30 casos si se quería tomar un control por cada caso, y por lo menos 27 casos si se quería tomar 4 casos por cada control. El presente estudio incluyó 33 casos y 112 controles.

Fuentes de los casos: Para depurar la información fue preciso recurrir a las siguientes fuentes:

1. Certificado de defunción de las muertes ocurridas en las mujeres en edad fértil.

2. Identificación de las muertes maternas debidas a causas obstétricas directas e indirectas.

3 . Registros de egresos hospitalarios por muertes correspondientes a todas las mujeres en edad fértil, ocurridas en todas las instituciones de salud públicas y privadas.

4. Informes de necropsias del Instituto de Medicina Legal de Medellín y de los hospitales del área. Se revisaron todos los informes de necropsia de las mujeres en edad fértil durante todo el período en estudio.

5. Historias clínicas de todas las muertes maternas y de las muertes de mujeres en edad reproductiva que habían sido clasificadas como "dudosas" tanto en la evaluación de los certificados de defunción como en la revisión de los registros de egresos hospitalarios y de los informes de necropsia durante el período en estudio.

6. Entrevista con los familiares más cercanos de las mujeres fallecidas y consideradas como casos y con los familiares de aquellas mujeres cuyo diagnóstico aparecía como dudoso para muerte materna.

7. Confirmación o eliminación de los casos considerados como "dudosos" después de revisar las diferentes fuentes de información.

Selección de los controles: Se tomaron dos controles institucionales y dos poblacionales por cada caso. Para seleccionar los controles poblacionales se utilizó el muestreo aleatorio estratificado teniendo en cuenta las comunas y los barrios, con base en la clasificación de Planeación Metropolitana. El $70.1 \%$ de la población pertenecía a Medellín y el $29.9 \%$ al resto del área. Para la selección de los controles institucionales se hizo un muestreo aleatorio sistemático teniendo en cuenta el número de muertes maternas ocurridas en cada una de las instituciones hospitalarias, con el fin de seleccionar los controles en forma proporcional a las mismas. Se tomaron como base los listados numerados de todos los egresos por abortos y partos ocurridos durante el período en estudio.

Las fuentes de información de los controles fueron los registros hospitalarios postparto y postaborto, las historias clínicas y las entrevistas con los familiares más allegados de las mujeres que habían sido seleccionadas como controles.

Procesamiento y análisis de la información: Los datos fueron procesados en microcomputador y se utilizó el programa Epi-info 5.0 de la Organización Mundial de la Salud y el Centro para el Control de Enfermedades Transmisibles de Atlanta (CDC). Las hipótesis básicas de trabajo corresponden a las de un estudio de casos y controles, en el que se plantea que la proporción de exposición a cada uno de los factores de riesgo en estudio es mayor en los casos (muertes maternas) que en los controles. Cuando las razones de disparidad son mayores que 1.0 se cumplen las hipótesis.

\section{Resultados y discusión}

Fuentes de informaciọn: El estudio de los certificados de defunción de las mujeres en edad fértil permitió conocer el $47.2 \%$ de las muertes maternas. Los informes de necropsia permitieron encontrar el $41.5 \%$ de las muertes y la revisión de las historias clínicas de las mujeres en edad fértil que murieron durante el período de estudio y que se produjeron tanto en los servicios de gineco-obstetricia, como en otros servicios de las instituciones de salud, permitó encontrar el 9.43\% de las muertes maternas. El subregistro encontrado fue del $52.8 \%$, debido probablemente a las fallas en las formas de llenar el certificado de defunción, porque la causa obstétrica es omitida la mayoría de las veces. Además, en tres de los casos no se encontró el certificado de defunción, probablemente porque las mujeres estudiadas fueron sepultadas en lugares diferentes al AMM.

Estructura de la Mortalidad en las Mujeres en Edad Fértil: La tasa de mortalidad en mujeres en edad fértil fue de 15 por 10.000 . La tercera parte de estas muertes se debió a causas violentas y accidentales $(27.7 \%$ y $6.7 \%$ respectivamente). La Mortalidad Materna fue responsable del 3.8\% de estas muertes, a diferencia de lo que ocurre en la mayoría de los países en desarrollo donde a la mortalidad materna se le atribuyen entre un tercio y un cuarto de todas las muertes de las mujeres en edad fértil.

La tasa de mortalidad por violencia fue de 4.2 por 10.000 mujeres en edad fértil. Esta tasa es comparable con las tasas de mortalidad general por homicidio en los primeros cinco años de la década de los 80 s, las cuales fluctuaron entre 4.2 y 10.1 por 10.000 y se presentaron fundamentalmente a expensas de la población masculina. Estos hallazgos están de acuerdo con la situación general del país, donde la contribución porcentual de los homicidios a la mortalidad general se ha ido incrementando desde un $3.51 \%$ en 1976 hasta $16.6 \%$ en 1985. En la ciudad de Medellín se ha evidenciado un incremento en el riesgo de muerte por homicidio en las mujeres que al final de la década llegó a ser cinco veces el del principio de la misma (2).

Este fenómeno de deterioro alcanza también a la mujer embarazada. Es así como en el presente estudio, de las 10 mujeres embarazadas que murieron por causas no obstétricas, siete muertes $(70 \%)$ fueron ocasionadas por arma de fuego, dos por accidente de tránsito $(20 \%)$ y una por quemaduras

Muertes durante el proceso reproductivo. La mayor proporción se debió a causas obstétricas directas $(71.7 \%)$. Casi la quinta parte de las muertes (18.9\%) se debió a causas no obstétricas, especialmente por trauma y violencia como ya se mencionó.

Mortalidad Materna. La tasa de Mortalidad Materna fue 7.19 por 10.000 nacidos vivos, más de dos veces la que informan los registros oficiales para 1988. Estos hallazgos concuerdan con otros estudios realizados en América Latina y El Caribe (3). En países desarrollados como los Estados Unidos se ha encontrado una proporción de muertes maternas que es superior en $32 \%$ a las cifras informadas por el Centro Nacional de Estadísticas de Salud (4). La mortalidad materna estimada para Colombia en 1986 fue de 11.0 por 10.000 nacidos vivos. En el presente estudio la tasa encontrada fue 2.05 veces la de los registros oficiales, lo cual hace suponer que la tasa de mortalidad materna en el país para dicho año 
pudo ser alrededor de 22.6 por 10.000 nacidos vivos.

De acuerdo con las cifras oficiales Colombia estaría ubicado dentro del grupo de países que la OPS ha calificado como de "alta mortalidad" (6.0-14.0 por $10.000 \mathrm{NV})$. Pero si se consideran las cifras reales de acuerdo con los hallazgos del presente estudio, el país estaría en la categoría de países con "muy alta mortalidad" (tasas superiores a 14.0 por 10.000 NV).

Estructura de la Mortalidad Materna. De las 33 muertes maternas ocurridas en el AMM, el $12.1 \%$ corresponden a muertes obstétricas indirectas. Esta proporción es mucho mayor que la informada para Colombia en 1985 que fue de $0.8 \%$. De las cuatro muertes por causas obstétricas indirectas, dos se presentaron durante el embarazo y dos en el postparto. Sin embargo, la proporción de muertes por causas obstétricas indirectas en el presente estudio está más relacionada con la búsqueda exhaustiva en otras fuentes de información, pues los certificados de defunción generalmente no mencionan el embarazo o las condiciones asociadas al mismo.

Dentro de las causas obstétricas directas, la hipertensión asociada al embarazo (síndrome pre-eclampsia eclampsia) fue la más frecuente ( $39.4 \%$ de las muertes), con una tasa específica de 27.0 por 100.000 nacidos vivos. Esta es una causa de muerte mucho más relievante de lo que demuestran las cifras oficiales, tanto en Colombia como en otros países de América Latina $(3,5)$. En el presente estudio la frecuencia no fue mayor que la de las cifras oficiales para el país (41.4\% en 1984), pero la tasa específica sí fue superior.

La segunda causa más frecuente fue la infección, con un $24.2 \%$ que equivale a una tasa de 6.2 por 100.000 nacidos vivos. Dentro de este rubro están consideradas 5 muertes por aborto y tres infecciones puerperales. Al considerar el número total de abortos que fueron 6 , la proporción de muertes maternas es del $18.2 \%$ y la tasa específica es de 1.2 por 100.000 nacidos vivos. El aborto había sido la segunda causa de muerte materna en Colombia en 1984, con una proporción del $23 \%$. Es conveniente tener en cuenta que si bien el subregistro de la Mortalidad Materna es alto, se acepta que éste es mucho mayor cuando la causa es el aborto, pues tanto en los países desarrollados como en desarrollo, la experiencia ha demostrado que la notificación de defunciones es casi siempre incompleta porque en el certificado de defunción muchas veces no se menciona el hecho de que la mujer había estado recientemente embarazada, lo cual es especialmente cierto cuando el embarazo había culminado en aborto.

Al discriminar las tres infecciones puerpuerales, la tasa específica es de 9.1 por 100.000 nacidos vivos.

La tercera causa fue la hemorragia, la cual se presentó en el $12.2 \%$ de los casos con una tasa de 8.1 por 100.000 nacidos vivos.

Dentro de las denominadas otras causas de muerte materna se encontró una debida complicación anestésica que corresponde a una tasa de 2.2 por 100.000 nacidos vivos.

\section{Análisis de los factores de riesgo asociados a la mortalidad materna.}

A. Socio-económicos y de condiciones de vida: Este grupo estuvo conformado por 9 factores de riesgo, en cinco de los cuales se encontró asociación con la mortalidad ma- terna y una tendencia lineal estadísticamente significativa. El gráfico 1 permite apreciar tanto las razones de disparidad como sus intervalos de confianza del $95 \%$ para estos cinco factores de riesgo. Al ordenarlos de acuerdo con la fuerza de asociación se encuentran de la siguiente manera:

1. Escolaridad de la mujer, máximo a nivel de primaria completa.

2. Pertenecer al estrato socio-económico bajo.

3. Ausencia del apoyo económico del esposo o compañero.

4. Ausencia del apoyo afectivo del esposo o compañero.

5. Escolaridad del esposo o compañero, máximo a nivel de primaria completa.

Gráfico 1

INTERVALOS DE CONFIANZA DEL 95\% DE RAZONES DE DISPARIDAD QUE TUVIERON SIGNIFICANCIA ESTADISTICA PARA LOS FACTORES DE RIESGO SOCIOECONOMICOS

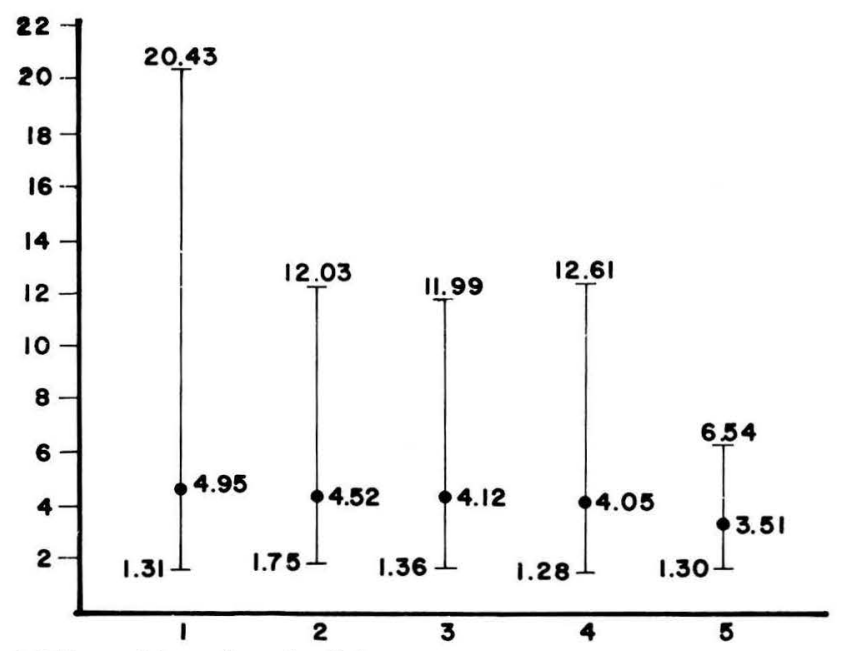

1 Educación primaria del esposo

2 Estrato socio económico bajo

3 Ausencia apoyo económico del esposo

4 Ausencia apoyo afectivo del esposo

5 Educación primaria de la mujer

Otros tres factores de riesgo que se consideró importante estudiar, pero en los cuales la asociación no fue estadísticamente significante fueron los siguientes:

- Ausencia de apoyo afectivo de la familia.

- Ocupación con predominio del trabajo físico.

- Hacinamiento.

La asociación entre los bajos niveles de escolaridad tanto de la mujer como de su compañero con la Mortalidad Materna se ha encontrado en otros estudios analíticos, como el realizado por Bhatia en la India (6), el cual encontró fuerte asociación especialmente con los controles del área rural. La relación entre la escolaridad y el estado de salud ha sido documentada en Colombia por el Estudio Nacional de Salud (1977-1980).

Las variables psicoafectivas apenas se empiezan a analizar en el campo de la Mortalidad Materna, aunque su importancia ha sido reconocida desde antes. 
La situación socio-económica fue evaluada tanto a través del estrato al cual pertenecía la mujer como a partir del apoyo económico recibido del esposo o compañero. Los riesgos respectivos fueron 4.5 para el estrato bajo y 4.0 para la ausencia del apoyo económico del esposo o compañero. Estos hallazgos son congruentes con la literatura disponible sobre Mortalidad Materna, en la cual se asocia la presencia de estas variables con las precarias condiciones de salud, por la cadena de efectos que originan en el proceso salud-enfermedad. Este hallazgo también es comparable con el estudio de la India, en el cual se encontró que los niveles de Mortalidad Materna de las áreas pobremente desarrolladas eran cuatro veces los de aquellas áreas con mayor desarrollo.

B. Relacionados con los antecedentes personales y obstétricos: En este grupo fueron estudiados trece factores de riesgo que fueron distribuidos de la siguiente manera: estado civil, edad (menor de 20 años y mayor de 30), paridad (nulípara, multípara -4 o más embarazos-), abortos previos $(1,2,1$ ó 2$)$, mortinatos previos, intervalo intergenésico, número de hijos vivos, antecedentes patológicos en embarazos anteriores, antecedentes quirúrgicos.

El único factor de riesgo de este grupo que presentó una asociación significativa con la Mortalidad Materna fue el estado civil, donde las mujeres solteras presentaron una probabilidad de muerte materna que era 2.6 veces la de aquellas que tenían un estado civil diferente, pero las hemorragias presentan una mayor fuerza de asociación aunque la razón de disparidad sea menor. (Gráfico 2).

Gráfico 2

ANTECEDENTES PERSONALES Y OBSTETRICOS RAZON DE DISPARIDAD

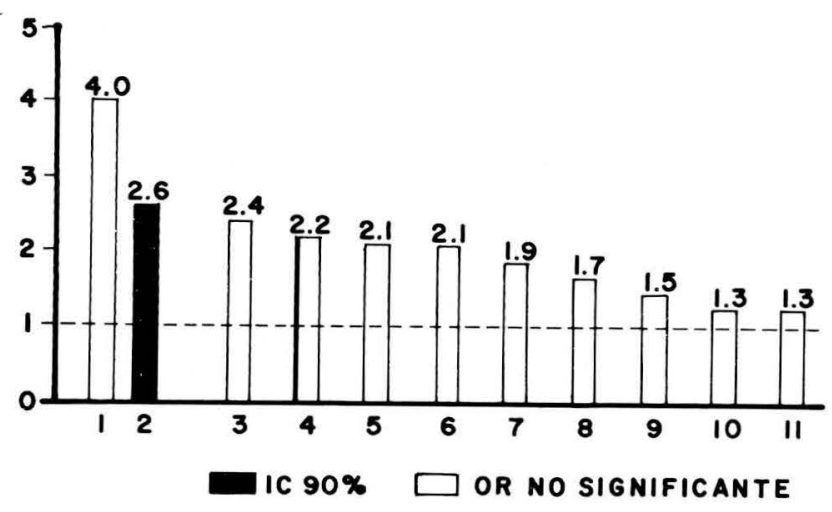

FACTORES DE RIESGO

1. Dos abortos previos.

2. Estado civil soltera.

3. Uno o dos abortos previos.

4. Más de cuatro embarazos.

5. Ultimo hijo menor de dos años.

6. Edad mayor de 30 años.

7. Un aborto previo.

8. Antecedentes patológicos en embarazos previos.

9. Mortinatos previos.

10. Primigestante.

11. Antecedentes quirúrgicos.

O.R. = Odds ratio: razón de disparidad.

I.C.: Intervalo de confianza en el que se encuentra la O.R.
C. Factores de riesgo del embarazo en el cual se produjo la muerte: Fueron estudiados diez factores de riesgo y sólo en seis de ellos se encontró asociación con la Mortalidad Materna. Tanto las razones de disparidad como los intervalos de confianza del $95 \%$ dentro de los cuales se encontraron dichas razones, pueden ser apreciadas en el gráfico 3. Al ordenar estos factores de riesgo de acuerdo con su fuerza de asociación se presentan de la siguiente manera.

1. Hemorragias.

2. Menos de 29 semanas al final del embarazo.

3. Cesárea.

4. Complicaciones en el puerpuerio.

5. Complicaciones en el trabajo de parto.

6. Hipertensión.

La hipertensión que fue la causa más frecuente de muerte, cuando se toma como factor de riesgo para la Mortalidad Materna, aparece como complicación del último embarazo con una razón de disparidad alta (10.01), es decir, su presencia aumenta 10 veces la probabilidad de morir en aquellas embarazadas que la padecen, en comparación con aquellas que no la sufren.

Gráfico 3

INTERVALOS DE CONFIANZA DEL 95\% DE RAZONES DE DISPARIDAD QUE TUVIERON SIGNIFICANCIA ESTADISTICA PARA LOS FACTORES DE RIESGO RELACIONADOS CON EL ULTIMO EMBARAZO

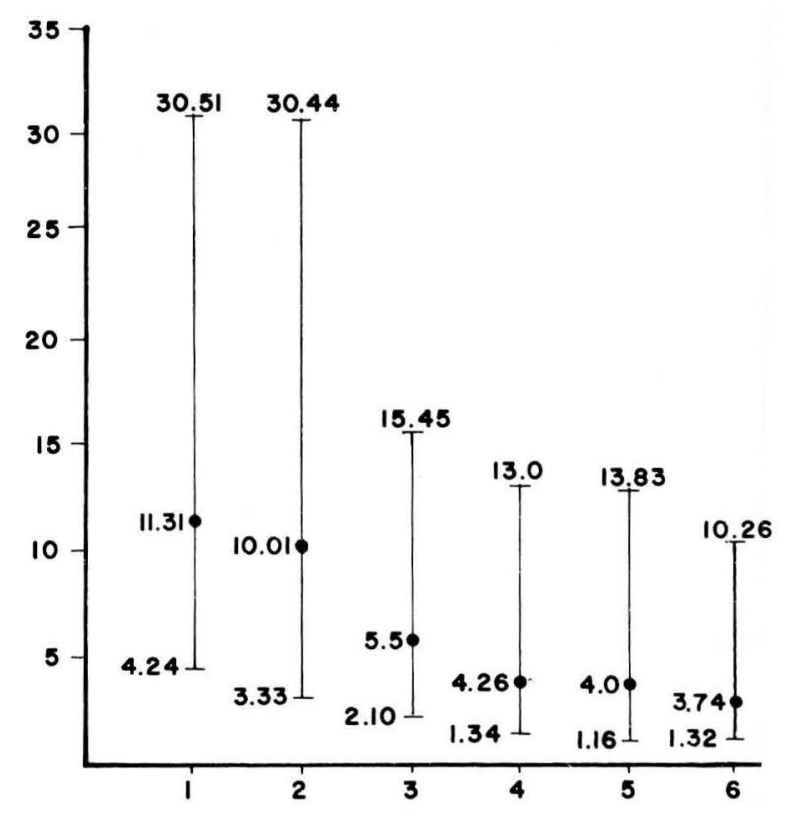

1 Complicaciones en el trabajo de parto

2 Hipertensión

3 Complicaciones del puerpuerio

4 < 29 semanas de amenorrea

5 Cesáreas

6 Hemorragias 
D. Factores de riesgo relacionados con la accesibilidad a los servicios de salud: Estos factores de riesgo pueden agruparse en los que tienen que ver con el control prenatal y los que se relacionaron con el lugar de atención del evento que condujo a la muerte.

Entre los factores que tienen que ver con el control prenatal se estudiaron los siguientes:

- Carencia de control prenatal.

- Consulta prenatal tardía (después de la décimo-segunda semana de amenorrea).

- Menos de cuatro controles prenatales.

El factor en el cual se encontró mayor fuerza de asociación fue tener menos de cuatro controles prenatales (gráfico 4), en el cual la asociación se encontró en el borde de la significancia.

En cuanto a los niveles de atención del evento que condujo a la muerte, se consideró como factor de riesgo la atención en el nivel primario con respecto a los demás niveles. Esta asociación no fue estadísticamente significativa.

\section{Gráfico 4}

ACCESIBILIDAD SERVICIOS DE SALUD

RAZONES DE DISPARIDAD

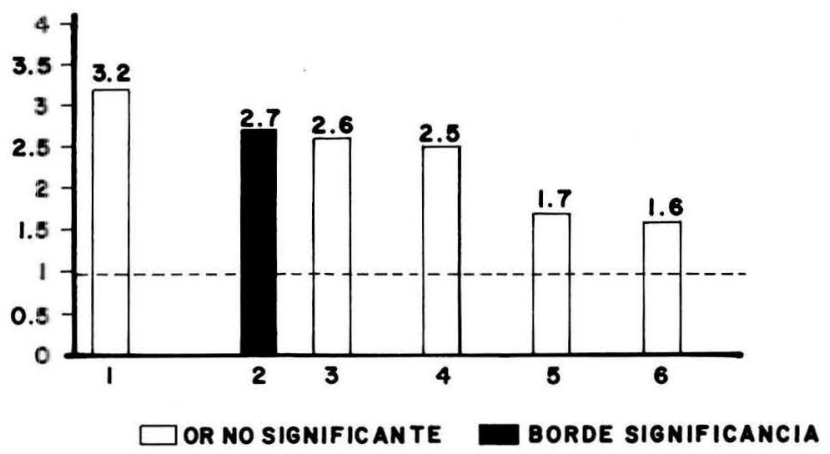

FACTORES DE RIESGO

1. Primera consulta después de las 20 semanas.

2. Menos de cuatro controles prenatales.

3. Atención en primer nivel comparada con el segundo.

4. Sin control prenatal.

5. Primera consulta entre las 12 y 20 semanas.

6. Atención en primer nivel comparada con segundo y tercero.

O.R. = Odds ratio: razón de disparidad.

\section{Evitabilidad de las muertes maternas}

Tradicionalmente la evitabilidad de las muertes maternas se ha enfocado a partir del estudio de caso o a partir de estudios descriptivos institucionales. El presente estudio analítico permitió obtener medidas del impacto mediante el cálculo de las Proporciones de Riesgo Atribuible a los Expuestos a los Factores de Riesgo, las cuales fluctuaron entre $61.5 \%$ y $91 \%$. Esto significa que si se pudieran eliminar los factores de riesgo en las mujeres expuestas a ellos, se podría evitar entre el $61.5 \%$ y el $91 \%$ de las muertes maternas en el AMM.

\section{Conclusiones}

- Para precisar el indicador de Mortalidad Materna se requieren otras fuentes de información que complementen la que se obtiene del certificado de defunción. De esta manera se pudo establecer una tasa de mortalidad materna que es más del doble de la informada oficialmente y una proporciốn de muertes obstétricas indirectas del $12.1 \%$.

- La Mortalidad Materna hace parte de la mortalidad de la mujer. Sin embargo, en el AMM el 27.7\% de las muertes de mujeres en edad reproductiva se debe a la violencia.

- El grupo de factores de riesgo de tipo socioeconómico presentó mayor asociación con la Mortalidad Materna en el AMM y se destacan especialmente la baja escolaridad en los dos miembros de la pareja, el estrato socioeconómico bajo y las escasas condiciones de apoyo afectivo para la mujer.

- El impacto sobre los factores de riesgo estudiados, especialmente sobre los de tipo socioeconómico, podría evitar entre el $61.5 \%$ y el $91 \%$ de las muertes maternas en el AMM.

- Los factores de riesgo relacionados con el embarazo en el cual se produjo la muerte materna pueden ser considerados como efectos de los factores de riesgo socioeconómicos y los relacionados con la accesibilidad a los servicios de salud.

\section{Recomendaciones}

1. Llevar a cabo intervenciones dentro del enfoque de riesgo con base en los hallazgos del presente estudio, con el fin de evitar o disminuir las muertes de las mujeres en edad reproductiva en el AMM. Algunas de estas intervenciones pueden ser las siguientes:

- Establecer un sistema de Vigilancia Epidemiológica sobre los factores de riesgo de la Mortalidad Materna, más que sobre el número de muertes maternas.

- Buscar la participación de otros sectores diferentes al sector salud para majorar el nivel educativo y el nivel de empleo de la población, especialmente en los sectores bajo y bajo-bajo.

- Organizar redes de apoyo psicológico para la mujer a nivel familiar y comunitario.

- Mejorar la cobertura y calidad de la atención a la mujer, especialmente durante su proceso reproductivo.

- Mejorar la calidad de las fuentes de información, en especial la de los certificados de defunción y las historias clínicas.

2. Realizar investigaciones operativas que permitan evaluar intervenciones específicas sobre algunos de los factores de riesgo que se encontraron asociados con la Mortalidad Materna en el presente estudio.

3. Llevar a cabo estudios semejantes en otras áreas de Colombia y América Latina, dada la gran semejanza que el AMM de Medellín guarda con ellas, en especial con el creciente proceso de urbanización que vive el subcontinente en el momento actual. Estos estudios permitirán la comparación no sólo en los hallazgos sino también en las intervenciones que podrían derivarse para lograr una disminución efectiva de la Mortalidad Materna y en consecuencia lograr un impacto en la salud de la mujer.

4. Trabajar sobre la Mortalidad Materna dando énfasis a su componente de Evitabilidad, para desarrollar indicadores positivos en la Salud de la Mujer. 


\section{BIBLIOGRAFIA}

1. Schlesselman JJ. Case-control studies. Design, conduct, analysis. Oxford University Press. pp. 145-150.

2. García HI., Vélez CH. Violencia en Medellín. Facultad Nacional de Salud Pública. Medellín, Colombia, 1990.

3. Walker G., Ashley D. Maternal Mortality in Jamaica. The Lancet, 1986; 1 (8479): 486-488.

4. Rochat RW. et al. Maternal Mortality in the United States: Report from the maternal mortality collaborative. Obstetrics and Gynecology 1988; Vol. 72 No. 1.

5. OPS/OMS. Programa de Salud Materno Infantil. Elementos básicos para el estudio y para la prevención de la mortalidad materna. Fascículos I y II. Washington, D.C. 1986.
6. Bhatia JC. A study o maternal mortality in Anantrapur district. Andhra Pradesh, India. Indian institute of Management. Sponsored by the WHO, 1986.

7. Cataño, LO., Cuervo F. El riesgo en la Mortalidad Materna. Boletín Epidemiológico de Antioquia. 1986; año XI, No. 2 Abril-Junio.

8. Organización Mundial de la Salud. Guía para el estudio de la Mortalidad Materna en los países en desarrollo. Tasas y causas. Ginebra, 1987.

9. Organización Mundial de la Salud. Mortalidad Materna: Ayudar a las mujeres a evitar el camino de la muerte. Crónicas de la OMS 1986; 40(5): 95-205. 